\title{
Viscous attenuation of a detonation wave propagating in a channel
}

\author{
P. Ravindran ${ }^{1}$, R. Bellini ${ }^{1}$, T.-H. Yi ${ }^{2}$, and F.K. Lu ${ }^{1}$ \\ 1 Aerodynamics Research Center, University of Texas at Arlington, Arlington, Texas, USA \\ 2 Institute of High Performance Computing, Singapore
}

Summary. The initiation and propagation of a detonation wave in a two-dimensional channel is simulated by an Euler and a Navier-Stokes solver. Transport processes were found to play a role in the wave propagation, resulting in a lower wave propagation speed arising from viscous drag.

\section{Introduction}

The propagation of a detonation wave remains one of the challenging problems in physics. While it is possible to predict the average propagation velocity almost correctly using classical theory $[1,2]$, there is no theory to explain the internal flow structure satisfactorily. The famous Zel'dovich-von Neumann-Döring theory $[3,4,5]$ is widely considered to describe the one-dimensional detonation structure correctly. Early experiments uncovered that the reduction to one space dimension is not entirely justified in long tubes. The detonation waves are non-planar and exhibit multi-dimensional substructures [18]. Numerical simulations have lately been used to examine these transient sub-structures but most of these studies thus have far neglected transport phenomena partly to simplify the approach. Moreover, neglecting transport phenomena appeared to be feasible in regions where such phenomena can be neglected. Nonetheless, the presence of walls requires careful consideration of transport phenomena [7].

Recent progress in computing power has enabled Navier-Stokes solutions of detonation waves $[8,9,10]$. A preliminary study on the effect of transport processes is reported here. The propagation of a detonation wave is examined by a comparison between an inviscid and a viscous solution.

\section{Governing Equations}

The governing equations for a high-temperature, chemically reacting viscous flow are modeled with thermodynamic processes and chemical reactions as well as the fluid dynamics. The gas considered is assumed to be thermally perfect and chemically nonequilibrium with finite-rate chemistry. For brevity, the derivations and relations are only summarized here; details can be found in [11]. The equations to be solved are the twodimensional, unsteady conservation equations extended for multi-species with chemical reactions. The species are assumed to be thermally perfect gas. Thus, the specific heats, enthalpy, entropy and internal energy of each species are functions of temperature only and are expressed as approximations by a least-squares fit method [12]. The expressions and coefficients are valid for $200-6000 \mathrm{~K}$ that exceeds the temperature range of interest. Dalton's law is used to obtain the pressure of the mixture from the partial pressures of the 
individual species. The overall reaction is that for a stoichiometric hydrogen-air mixture. The elementary reaction mechanisms for the mixture are extracted from the GRI-Mech 3.0 [13] database that contains 325 reactions and 53 species. From this database, the 9species 28-reaction model was chosen. The transport properties and third body reaction data were also extracted from the GRI-Mech 3.0 database.

The viscosity coefficient, diffusion coefficient and thermal conductivity were obtained from classical kinetic theory [14]. The viscosity coefficient and thermal conductivity of species are independent of the pressure whereas the diffusion coefficient is inversely proportional to the pressure. It is noted that the mixture-averaged formulations for the thermal conductivity and diffusion coefficient are only approximations in order to reduce computational time [14].

\section{Numerical Formulation}

The two-dimensional Navier-Stokes equations were discretized by a finite volume formulation and retaining the integral form with the source term. The convective and viscous fluxes were evaluated at the faces of a cell in the $x$ - and $y$-direction. The convective fluxes were approximated by flow quantities extrapolated to the left and right sides of the cell face while the viscous fluxes were computed by the averaged variables at a face and then were approximated using a second-order Roe scheme which was extended for a multi-species, thermally perfect and non-equilibrium gas $[15,16]$. High-order accuracy in space is achieved by using the MUSCL (Monotone Upstream-Centered Schemes for Conservation Laws) approach. By employing an appropriate minmod limiter, the MUSCL scheme becomes first-order accurate in the vicinity of the discontinuities and secondorder accurate in smooth regions. For temporal discretization, the governing equations were discretized in time so that they become a system of ordinary differential equations. A two-step Runge-Kutta scheme was utilized to achieve second-order accuracy. Since this scheme is fully explicit, it can be started with known values at time level $n$. The transport terms were evaluated using Green's theorem [17], but the evaluation of the source terms was much more complex due to the chemical reactions having much shorter time scales than those associated with the flow, resulting in stiffness. The approach used to overcome this difficulty is highlighted in [11]. The temperature must be evaluated at each grid cell whenever the species density was updated for a thermally perfect gas. Likewise, the boundary conditions were evaluated for the wall, supersonic inflow into the channel and outflow.

\section{Results}

A $50 \mathrm{~cm}$ long and $10 \mathrm{~cm}$ high channel was used for the detonation channel and the computational domain was discretized into $100 \times 150$ grid cells. The grids were clustered near the walls to capture the boundary layer. Adiabatic wall boundary conditions were imposed on the upper and lower sides of the computational domain while supersonic inflow and outflow were imposed on the left and right side respectively, with an ambient pressure of $1 \mathrm{~atm}$ imposed at the outlet of the channel. Pre-mixed hydrogen-air gas at an ambient pressure of $2 \mathrm{~atm}$ and temperature of $500 \mathrm{~K}$ was set to flow into the channel at an incoming Mach number of 2 . The mixture was ignited by a localized hot spot at 


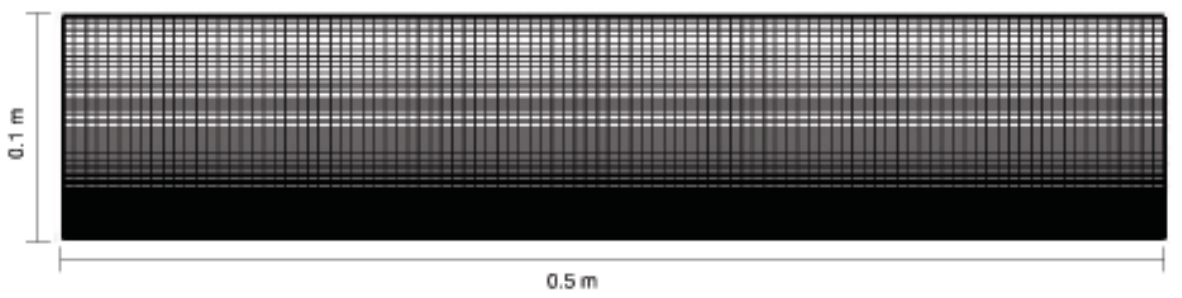

Fig. 1. Schematic of the computational domain

approximately $0.1 \mathrm{~m}$ into the channel from the left end. The hot spot was located at the bottom of the channel. It was $1 \mathrm{~mm}$ wide and $5 \mathrm{~mm}$ high. The pressure and temperature in the hot spot were 30 atm and $3000 \mathrm{~K}$ respectively. Detonation waves were immediately generated and the initiation and propagation are shown in the results.
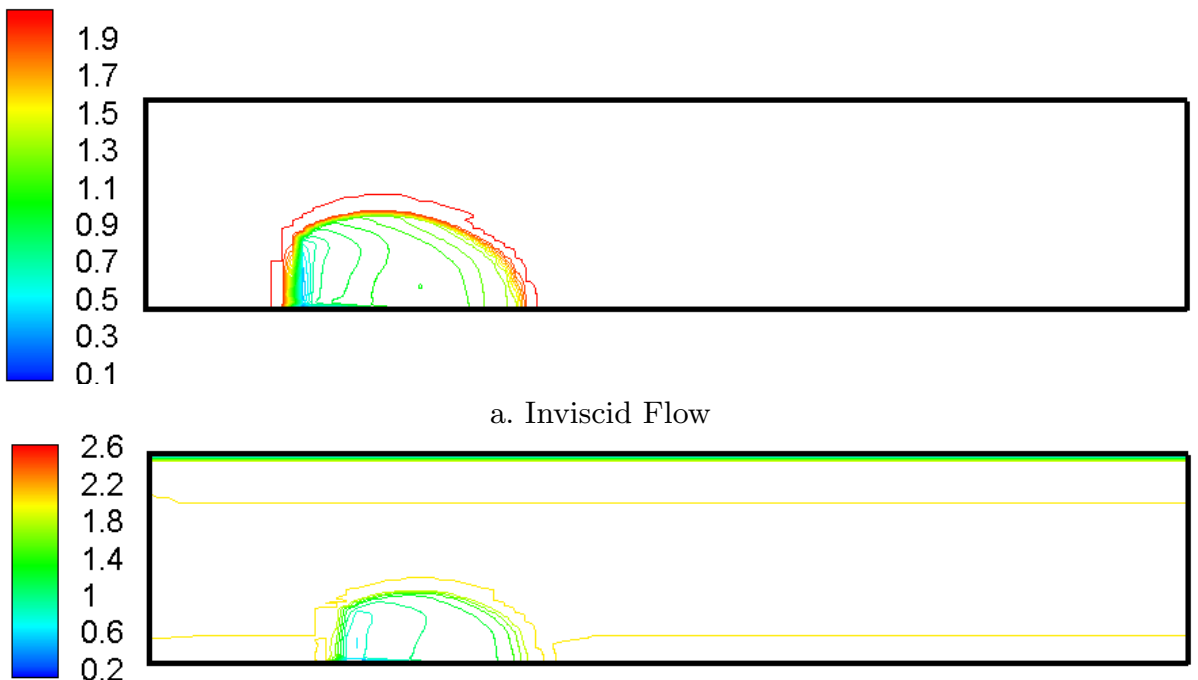

b. Viscous Flow

Fig. 2. Mach number distribution: detonation initiation snapshot at $t=1.8090 \times 10^{-5} \mathrm{~s}$

The results of the numerical solution were compared with that of an inviscid algorithm to characterize the effect of viscosity on the flow. Figure 2 shows the initiation of the detonation wavefront at time $t=1.8090 \times 10^{-5} \mathrm{~s}$. Figure 3 indicates that the propagation of the wavefront at $t=5.9636 \times 10^{-5} \mathrm{~s}$ is almost half the length of the channel and Fig. 4 shows the wavefront exiting from the channel at $t=1.0107 \times 10^{-4} \mathrm{~s}$. Results in Figs. 5and 6 for the pressure and temperature, respectively, taken at $t=5.9636 \times 10^{-5} \mathrm{~s}$, show a similar pattern in wavefront propagation.

A comparison of the viscous and inviscid results shows that the wavefront propagation is delayed or attenuated due to the presence of viscosity. This can be attributed to the drag arising from the formation of a laminar boundary layer behind the shock wave $[18,19]$. Also, the pressure and temperature at the wall are found to be higher for viscous solutions, due to the presence of shear layers. The simulation also shows that the 

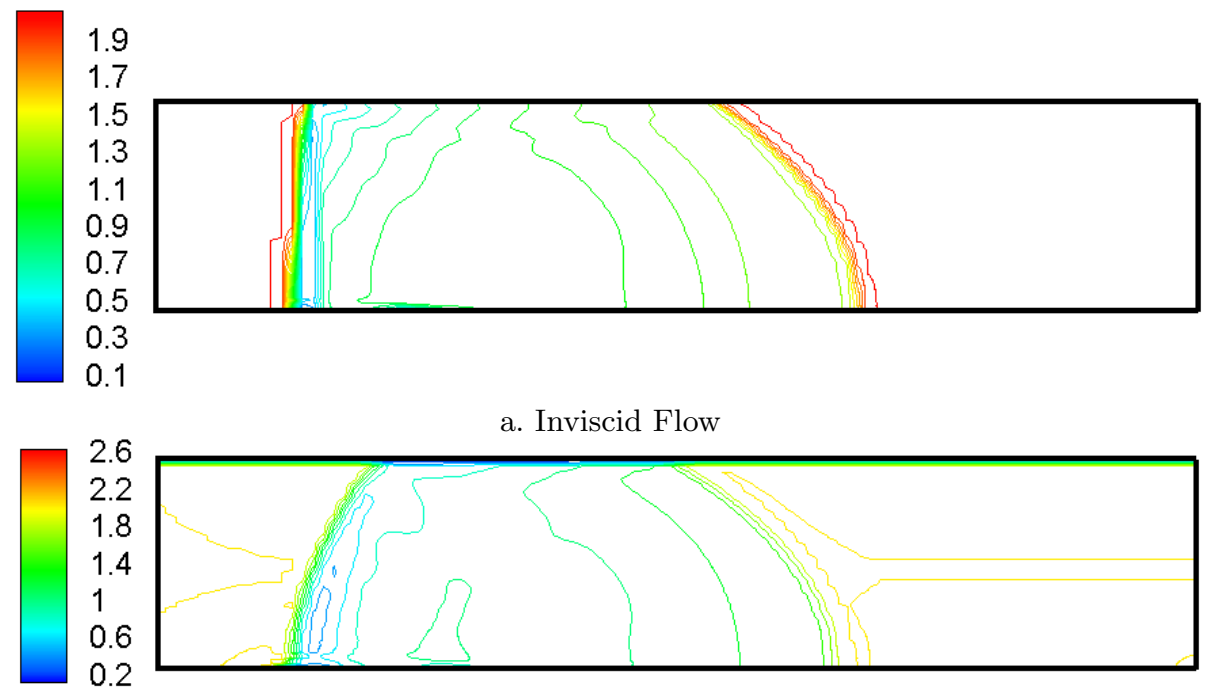

b. Viscous Flow

Fig. 3. Mach number distribution: detonation propagation snapshot at $t=5.9636 \times 10^{-5} \mathrm{~s}$

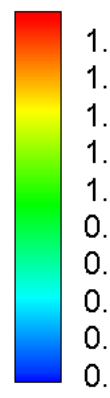

1.9

1.7

1.5

1.1

0.9

0.7

0.5

0.3

0.1

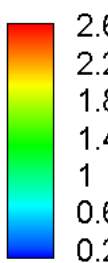

a. Inviscid Flow

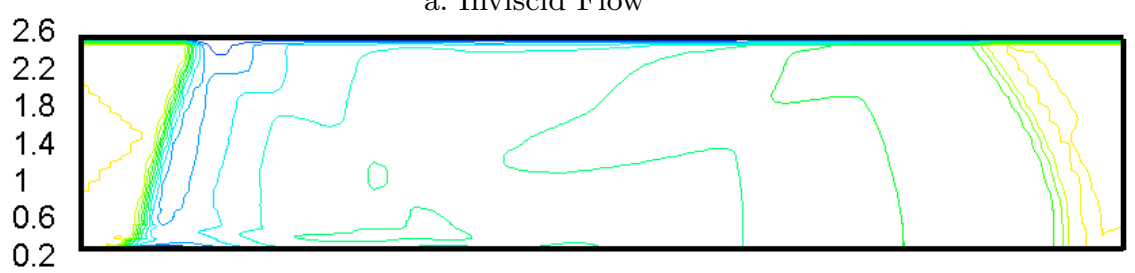

b. Viscous Flow

Fig. 4. Mach number distribution: detonation propagation snapshot at $t=1.0107 \times 10^{-4} \mathrm{~s}$

wave strength of the downstream moving detonation is significantly stronger than that of the upstream-moving detonation due to the non-zero Mach inflow condition. Another significant difference between the viscous and inviscid flows is observed in the downstream moving wavefront. For an inviscid simulation it was found to be a straight shock but the effect of transport processes is evident in the viscous simulation, wherein the downstream shock in more curved due to the velocity gradient from the walls to freestream. 


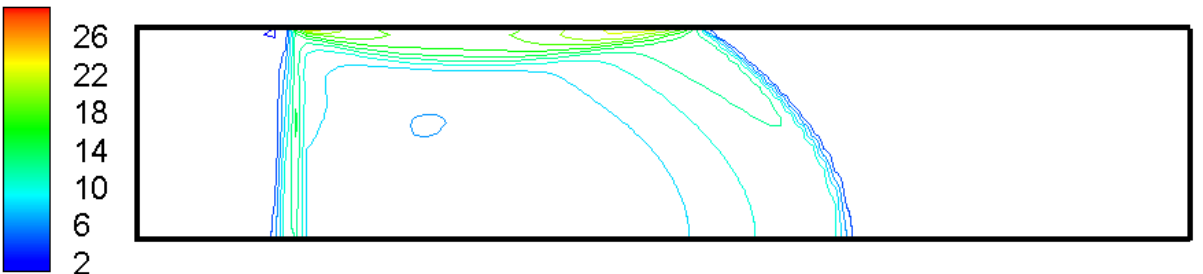

a. Inviscid Flow

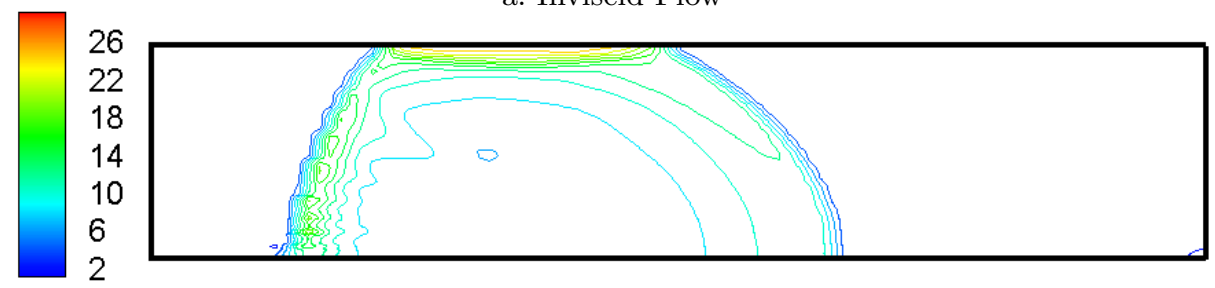

b. Viscous Flow

Fig. 5. Pressure distribution: detonation propagation snapshot at $t=1.8090 \times 10^{-05} \mathrm{~s}$

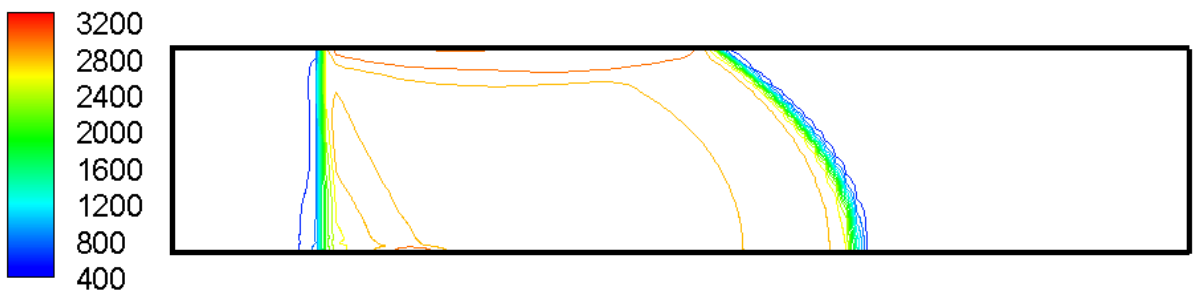

a. Inviscid Flow

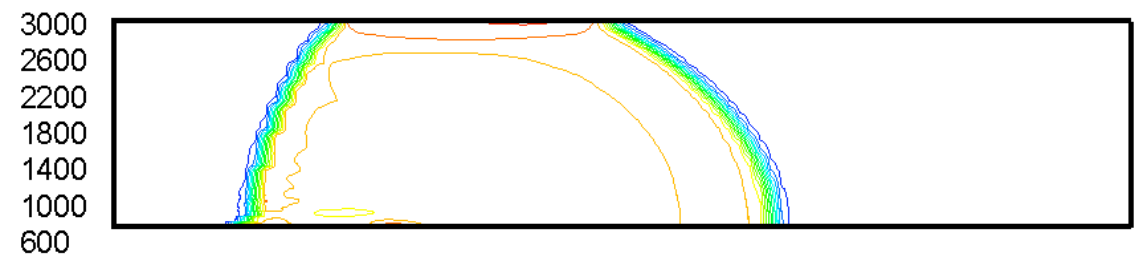

b. Viscous Flow

Fig. 6. Temperature distribution: detonation propagation snapshot at $t=1.8090 \times 10^{-5} \mathrm{~s}$

\section{References}

1. Chapman, D.L.: On the rate of explosion in gases. In: Philos. Mag., vol 47, pp 90-104, 1899

2. Jouguet E.: On the propagation of chemical reactions in gases. In: J. de Mathematiques Pures et Appliquees, vol. 1, pp. 347-425, 1906

3. Zel'dovich, Ya.B.: On the theory of the propagation of detonations on gaseous system. In: Zh. Eksp. Teor. Fiz., vol. 10, pp. 542-568, 1940

4. von Neumann, J.: Theory of detonation waves. Progress Report to the National Defense Research Committee Div. B, OSRD-549, (April 1, 1942. PB 31090); In: Taub, A. H. (ed). John von Neumann: Collected Works, 1903-1957, Vol. 6, Pergamon Press, New York, 1963

5. Döring, W.: On detonation processes in gases. In: Ann. Phys., vol. 43, pp. 421-436, 1943

6. Pintgen, F., Eckett, C.A., Austin, J.M., Shepard, J.E.: Direct observations of reaction zone structure in propagating detonations. In: Combust. Flame, vol. 133(3), pp. 221-229, 2003 
7. Vitello, P., Souers, P.C.: Stability effects of artificial viscosity in detonation modelling. In: 12th Int. Detonation Symp., San Diego, California, Aug 11-16, 2002

8. Oran, E.S., Gamezo, V.N.: Origins of the deflagration-to-detonation transition in gas phase combustion. In: Combust. Flame, vol. 148(1-2), pp. 4-47, 2007

9. Akkerman, V., Bychkov, V., Petchenko, A., Eriksson, L.-E.: Accelerating flames in cylindrical tubes with nonslip at the walls. In: Combust. Flame, vol. 145(1-2), pp. 206-219, 2007

10. Singh S., Rastigejev Y., Paolucci S., Powers J.M.: Viscous detonation in $\mathrm{H}_{2}-\mathrm{O}_{2}$-Ar using intrinsic low-dimensional manifolds and wavelet adaptive multilevel representation. In: Combust. Theory Modelling, vol. 5(2), pp. 163-184, 2000

11. Yi, T.-H.: Numerical study of chemically reacting viscous flow relevant to pulsed detonation engines. Ph.D. dissertation, Univ. Texas at Arlington, 2005

12. Gordon, S. and McBride, B.J.: Computer program for calculation of complex chemical equilibrium compositions and application I. Analysis. Tech. Rep. NASA RP-1311, 1976

13. Smith, G.P., Golden, D.M., Frenklach, M., Moriarty, N.W., Eiteneer, B.: GRI-Mech 3.0, [Online] Available: http://www.me.berkeley.edu/gri-mech

14. Kee, R.J., Coltrin, M., and Glarborg, P.: Chemically Reacting Flow, Wiley Interscience, 2003

15. Grossmann, B., and Cinella, P.: Flux-split algorithms for flows with nonequilibrium chemistry and vibrational relaxation. In: J. Comp. Phys., vol. 88, pp. 131-168, 1990

16. Deiterding, R.: Parallel adaptive simulation of multi-dimensional detonation structures. Ph.D. dissertation, Brandenburgische Technische University, Cottbus, Germany, 2003

17. Hirsch, C.: Numerical Computation of Internal and External Flows, Wiley, 1990

18. Liu, W.S., Du, X.X., and Glass, I.I.: Laminar boundary layers behind detonation waves. In: Proc. Roy. Soc. London. Ser. A, Math. Phys. Sci., vol. 387(1793), pp. 331-349, 1983

19. Zhang, F., Chue, R.S., Frost, D.L., Lee, J.H.S., Thibault, P., and Yee, Y.: Effects of area change and friction on detonation stability in supersonic ducts. In: Proc. Roy. Soc.: Math. Phys. Sci., vol. 449(1935) pp. 31-49, 1995 